\title{
Receding hopes of AIDS vaccines
}

\section{Prospects that there will soon be an effective vaccine against HIV are diminishing. In the absence of a good animal model of AIDS, direct human trials of candidate vaccines are inevitable.}

\section{Stockholm}

IT would have been extraordinary if the first shots at making an AIDS vaccine had been effective, so there need not be too much despondency now that they seem likely to fail. Not that they have failed yet in human beings; indeed, the trials have only just got under way. But the lack of success of initial trials in chimpanzees and rhesus monkeys cast a dark shadow over the IVth International Conference on AIDS, held here last week.

Pessimism tends to take over when someone like Gordon Ada, who is currently with the World Health Organization and has a wealth of experience in viral vaccines, says that the problems of producing a vaccine against the human immunodeficiency virus (HIV) are greater than for any other virus. Justifying that statement at the end of the conference, Ada listed a string of reasons. One is that HIV is notoriously variable. No two isolates are identical. Each isolate contains many variants. Variation may be HIV's way of escaping destruction by the immune system, and may yield variants of increasing pathogenicity and preferences for one tissue over another in the course of disease. A successful vaccine would have to be able to deal with a wide and shifting variety of strains.

A second serious problem is that the virus ducks for cover shortly after infection. That is to say, once HIV has infected a cell, its RNA genome is transcribed into DNA by reverse transcription, which then becomes integrated into the chromosomal DNA of the infected cell. An effective vaccine would have to prevent the virus from infecting any cells, for no vaccine can attack the integrated DNA. Viruses subsequently made under the instructions of that DNA will be susceptible to attack if they leave the shelter of the cell. But there is growing evidence that some cells may transmit their HIVs directly to other cells, without releasing them. To make matters worse, it may be that the original infecting virus is largely within cells in the blood, semen or vaginal fluids, rather than free, and is also transmitted from cell to cell. In that case a vaccine would never get sight of $\mathrm{HIV}$ in the first place.

Had all this been known three years ago, when the first candidate vaccines were on the drawing board, it might have influenced their design. As it was they were hurriedly designed from what was most available at the time. In essence, that meant the envelope protein of the virus. This forms the external surface of the virus and therefore is most easily seen by the immune system. It is also the protein that varies most between strains.

At the latest count no chimpanzee vaccinated with the envelope protein has been protected against infection by HIV. This is true whether the vaccine is made from the protein itself, or whether it consists of vaccinia virus, the smallpox vaccine, genetically engineered to contain the gene for HIV's envelope protein. The potential advantage of vaccines of this type is that live viruses activate parts of the immune system that proteins alone cannot reach. In doing so, they stimulate the production of immune cells that can recognize and kill virus-infected cells, which may well be a more important defence system than the production of antibodies.

Because of the shortage of chimpanzees, it has not been possible to ring the various changes that might turn the initial failures into success. What makes it questionable whether any easy change in the vaccine will make a difference is the failure of a trial of passive immunization described by Jorg Eichberg of the Southwest Foundation for Medical Research in Texas. Chimpanzees were infused with large quantities of immunoglobulin purified from the blood of AIDS patients. As a result they were briefly extremely well armed not only with antibodies against a range of HIV proteins, but also antibodies that neutralize the virus and antibodies that prevent the virus from fusing cells together. Yet, when challenged 24 hours later with HIV, they were infected.

Despite, or perhaps because of, these failures, four starts on vaccinating human beings have begun. One uses envelope protein alone. Made by MicroGeneSys, the protein has been given in four different doses to homosexual volunteers, some of whom received a booster shot one month later. The higher doses sometimes, but not reliably, induced antibody production and a positive lymphocyte blast transformation test. A yet higher dose, 180 micrograms, will now be tested.

Another vaccine, described recently in Nature $(322,728 ; 1988)$ uses both an engineered vaccinia virus and cells taken from the vaccinated subject infected with virus, killed and given back by slow infusion. Daniel Zagury, who has used himself as a guinea pig for this complex procedure, is now seeking a simplified version. In a third trial, with very tentative beginnings, two patients in London have been given an (anti-idiotypic) antibody designed to prevent infection by HIV.

Master-minded by Jonas Salk, the fourth trial involves giving killed HIV to people already infected with the virus in an attempt to boost their immune reaction against it. The virus, which is killed by gamma irradiation and then purified with the loss of envelope protein, was first given to nine people with AIDS related complex - the condition that precedes full-blown AIDS - last November. Salk reported just a hint of benefit in the results from this group so far. A second group of nine people joined the trial in March and 54 matched pairs of infected but asymptomatic volunteers have been recruited for the third stage.

The killed virus preparation has also been given to one uninfected and two HIV-1 infected chimpanzees, but they have yet to be tested for resistance to infection or reinfection. That humans greatly outnumber chimpanzees in the trials is a measure of the shortage of the latter. Moreover, chimpanzees do not develop AIDS after infection. For both reasons the hunt for an alternative animal model is intense. Since some simian immunodeficiency viruses (SIV) will produce AIDS in rhesus monkeys, this is a promising, if inexact model.

Unfortunately, the first attempt to vaccinate rhesus monkeys against SIV infection, using an inactivated SIV vaccine at the New England Primate Center, has failed. Another hope is that HIV-2, the predominant AIDS virus in western Africa, will infect rhesus monkeys and produce AIDS, even though HIV-1 will do neither. Groups in France and Sweden have begun to test this possibility and seem confident it will work on the basis of early signs. Finally there is a hint from separate laboratories in the United States and Italy that rabbits can be infected by HIV-1.

In the absence of a simple and reliable animal model for AIDS it is inevitable that pressures will mount for more exploratory trials of candidate AIDS vaccines in atrisk, or HIV-infected, volunteers. Such trials will need careful scrutiny before approval and should not be implemented without fully informed consent as they are not without imaginable risk. But there is little option other than to allow the best of them to proceed.
Peter Newmark 